\title{
On Faintly Continuous Functions via Generalized Topology
}

\author{
Bishwambhar Roy \\ Department of Mathematics, Women's Christian College, 6 Greek Church Row, Kolkata 700 026, India \\ Correspondence should be addressed to Bishwambhar Roy; bishwambhar_roy@yahoo.co.in
}

Received 13 July 2013; Accepted 9 September 2013

Academic Editors: Y. Ouyang, G. Wang, and W. Zhu

Copyright (C) 2013 Bishwambhar Roy. This is an open access article distributed under the Creative Commons Attribution License, which permits unrestricted use, distribution, and reproduction in any medium, provided the original work is properly cited.

The notion of faintly $(\mu, \sigma)$-continuous function has been introduced. Relationship between this new class of function with similar types of functions has been given. Some characterizations and properties of such function are also being discussed.

\section{Introduction}

In topology, weak forms of open sets play an important role in the generalization of different forms of continuity. Using different forms of open sets, many authors have introduced and studied various types of continuity. In this paper, a unified version of some types of continuity has been introduced from a generalized topological space to a topological space. Generalized topology was first introduced by Császár (see [15]).

We recall some notions defined in [1]. Let $X$ be a nonempty set, and $\exp X$ denotes the power set of $X$. We call a class $\mu \subseteq \exp X$ a generalized topology [1], (GT) if $\varnothing \epsilon$ $\mu$ and union of elements of $\mu$ belongs to $\mu$. A set $X$, with a GT $\mu$ on it, is said to be a generalized topological space (GTS) and is denoted by $(X, \mu)$. Let $(X, \tau)$ be a topological space. The $\delta$-closure [6] of a subset $A$ of a topological space $(X, \tau)$ is defined by $\{x \in X: A \cap U \neq \varnothing$ for all regular open set $U$ containing $x$, where a subset $A$ is called regular open if $A=\operatorname{int}(\operatorname{cl}(A))$. A subset $A$ of a topological space $(X, \tau)$ is called semiopen [7] (resp., preopen [8], $\alpha$-open [9], $\beta$-open [10], $b$-open [11], $\delta$-preopen [12], $\delta$-semiopen [13], and $e$-open [14]) if $A \subseteq \operatorname{cl}(\operatorname{int}(A))$ (resp., $A \subseteq \operatorname{int}(\operatorname{cl}(A)), A \subseteq$ $\operatorname{int}(\operatorname{cl}(\operatorname{int}(A))), A \subseteq \mathrm{cl}(\operatorname{int}(\operatorname{cl} A)), A \subseteq \mathrm{cl}(\operatorname{int}(A)) \cup \operatorname{int}(\operatorname{cl}(A))$, $A \subseteq \operatorname{int}\left(\mathrm{cl}_{\delta}(A)\right), A \subseteq \operatorname{cl}\left(\operatorname{int}_{\delta}(A)\right)$, and $A \subseteq \operatorname{int}\left(\operatorname{cl}_{\delta}(A)\right) \cup$ $\left.\operatorname{cl}\left(\operatorname{int}_{\delta}(A)\right)\right)$. A point $x \in X$ is in $\operatorname{scl}(A)$ (resp., $\left.p \operatorname{cl}(A)\right)$ if for each semiopen (resp., preopen) set $U$ containing $x, U \cap A \neq \varnothing$. A point $x \in X$ is called a $\theta$-cluster [6] (resp., semi- $\theta$-cluster [15], $p(\theta)$-cluster [16]) point of $A$ denoted by $\mathrm{cl}_{\theta}(A)$ (resp., $\left.s \mathrm{sl}_{\theta}(A), p(\theta)-\operatorname{cl}(A)\right)$ if $\operatorname{cl}(A) \cap U \neq \varnothing$ (resp., $s \mathrm{cl}(A) \cap U \neq \varnothing$, $\operatorname{pcl}(A) \cap U \neq \varnothing)$ for every open (resp., semiopen, preopen) set
$U$ containing $x$. A subset $A$ is called $\theta$-closed (resp., semi- $\theta$ closed, $p(\theta)$-closed) if $\operatorname{cl}_{\theta}(A)=A$ (resp., $s \operatorname{cl}_{\theta}(A)=A, p(\theta)$ $\operatorname{cl}(A)=A$ ). The complement of a $\theta$-closed (resp., semi- $\theta$ closed, $p(\theta)$-closed) set is called $\theta$-open (resp., semi- $\theta$-open, $p(\theta)$-open). The family of all $\theta$-open sets in a topological space forms a topology which is weaker than the original topology. The finite union of regular open sets is said to be $\pi$-open [17]. A subset $A$ of a topological space $(X, \tau)$ is said to be $\pi g$-closed [17] if $\operatorname{cl}(A) \subseteq U$ whenever $A \subseteq U$ and $U$ is $\pi$-open. A subset $A$ of $X$ is called $\omega$-open [18] if for each $x \in A$ there exists an open set $U$ containing $x$ such that $|U \backslash A| \leq \aleph_{0}$. The family of all $\omega$-open subsets of a space $(X, \tau)$ forms a topology on $X$ finer than $\tau$. For any topological space $(X, \tau)$, the collection of all semiopen (resp., preopen, $\alpha$-open, $\beta$-open, $b$-open, $\delta$-preopen, $\delta$-semiopen, $e$-open, $\theta$ open, semi- $\theta$-open, $p(\theta)$-open, $\pi$-open, and $\omega$-open) sets are denoted by $S O(X)$ (resp., $P O(X), \alpha O(X), \beta O(X), B O(X)$, $\delta P O(X), \delta S O(X), e O(X), \theta O(X), S_{\theta} O(X), P_{\theta} O(X), \pi g O(X)$, and $\omega O(X))$. We note that each of these collections forms a GT on $(X, \tau)$.

For a GTS $(X, \mu)$, the elements of $\mu$ are called $\mu$-open sets and the complement of $\mu$-open sets are called $\mu$-closed sets. For $A \subseteq X$, we denote by $c_{\mu}(A)$ the intersection of all $\mu$-closed sets containing $A$, that is, the smallest $\mu$-closed set containing $A$, and by $i_{\mu}(A)$ the union of all $\mu$-open sets contained in $A$, that is, the largest $\mu$-open set contained in $A$ (see $[1,2]$ ).

It is easy to observe that $i_{\mu}$ and $c_{\mu}$ are idempotent and monotonic, where $\gamma: \exp X \rightarrow \exp X$ is said to be idempotent if and only if $A \subseteq B \subseteq X$ implies $\gamma(\gamma(A))=\gamma(A)$ and monotonic if and only if $\gamma(A) \subseteq \gamma(B)$. It is also well known from $[2,3]$ that if $\mu$ is a GT on $X$ and $A \subseteq X, x \in X$, then 
$x \in c_{\mu}(A)$ if and only if $x \in M \in \mu \Rightarrow M \cap A \neq \varnothing$ and $c_{\mu}(X \backslash A)=X \backslash i_{\mu}(A)$.

Hereafter, throughout the paper, we will use $(X, \mu)$ to mean a generalized topological space and $(Y, \sigma)$ to be a topological space unless otherwise stated.

\section{Faintly $(\mu, \sigma)$-Continuous and Related Functions}

Definition 1. A function $f:(X, \mu) \rightarrow(Y, \sigma)$ is said to be faintly $(\mu, \sigma)$-continuous at $x$ if for each $\theta$-open set $V$ in $Y$ containing $f(x)$ there exists $U$ in $\mu$ containing $x$ such that $f(U) \subseteq V$. If $f$ is faintly $(\mu, \sigma)$-continuous at each point of $X$, then $f$ is called faintly $(\mu, \sigma)$-continuous on $X$.

Definition 2. A function $f:(X, \mu) \rightarrow(Y, \sigma)$ is said to be $(\mu$, $\sigma)$-continuous [19] (resp., weakly $(\mu, \sigma)$-continuous, almost $(\mu, \sigma)$-continuous) if for each $x \in X$ and each open set $V$ of $Y$ containing $f(x)$ there exists $U \in \mu$ containing $x$ such that $f(U) \subseteq V$ (resp., $f(U) \subseteq \operatorname{cl}(V), f(U) \subseteq \operatorname{int}(\operatorname{cl}(V)))$.

Remark 3. (i) Let $(X, \tau)$ and $(Y, \sigma)$ be two topological spaces. If $\mu=\tau, S O(X), P O(X), \alpha O(X), \beta O(X), B O(X)$, $\delta P O(X), \delta S O(X), e O(X), \theta O(X), S_{\theta} O(X), P_{\theta} O(X), \pi g O(X)$, and $\omega O(X)$, then a faintly $(\mu, \sigma)$-continuous function $f$ : $(X, \mu) \rightarrow(Y, \sigma)$ reduces to a faintly continuous [20], faintly semicontinuous [21], faintly precontinuous [21], faintly $\alpha$ continuous $[22,23]$, faintly $\beta$-continuous [21], faintly $\gamma$-continuous [21], faintly $\delta$-precontinuous [24], faintly $\delta$-semicontinuous [25], faintly $e$-continuous [26], quasi- $\theta$-continuous [21], faintly semi- $\theta$ continuous [27], faintly pre- $\theta$-continuous [27], faintly $\pi g$-continuous function [28], and faintly $\omega$-continuous function [29], respectively. On the other hand, every faintly $m$-continuous function [30] $f:\left(X, m_{X}\right) \rightarrow(Y, \sigma)$ is faintly $\left(m_{X}, \sigma\right)$-continuous if $m_{X}$ is closed under arbitrary union (as $m_{X}$ is a GT in that case).

(ii) It follows from Definitions 1 and 2 that

(a) every $(\mu, \sigma)$-continuous function is faintly $(\mu, \sigma)$-continuous;

(b) every $(\mu, \sigma)$-continuous function is almost $(\mu, \sigma)$ continuous;

(c) every almost $(\mu, \sigma)$-continuous function is weakly $(\mu$, $\sigma)$-continuous.

But the converses are false as shown in the next example.

Example 4. (a) Let $X=Y=\{a, b, c\}$, let $\mu=\{\varnothing,\{a, b\},\{b, c\}$, $X\}$, and let $\sigma=\{\varnothing,\{a\},\{a, b\}, X\}$. Consider the function $f$ : $(X, \mu) \rightarrow(Y, \sigma)$ defined by $f(a)=a, f(b)=b$, and $f(c)=a$. It is easy to check that $f$ is faintly $(\mu, \sigma)$-continuous but not $(\mu, \sigma)$-continuous.

(b) Let $X=Y=\{a, b, c\}$, let $\mu=\{\varnothing,\{a\},\{a, b\},\{b, c\}, X\}$, and let $\sigma=\{\varnothing,\{a\},\{c\},\{a, c\},\{b, c\}, X\}$. Consider the identity map $f:(X, \mu) \rightarrow(Y, \sigma)$. It can be easily checked that $f$ is almost $(\mu, \sigma)$-continuous but not $(\mu, \sigma)$-continuous.

(c) Let $X=Y=\{a, b, c\}$, let $\mu=\{\varnothing,\{a\},\{a, b\},\{b, c\}, X\}$, and let $\sigma=\{\varnothing,\{a\},\{c\},\{a, c\}, X\}$. Consider the identity map
$f:(X, \mu) \rightarrow(Y, \sigma)$. It can be easily checked that $f$ is not almost $(\mu, \sigma)$-continuous but weakly $(\mu, \sigma)$-continuous.

Theorem 5. For a function $f:(X, \mu) \rightarrow(Y, \sigma)$, the following statements are equivalent:

(a) $f$ is faintly $(\mu, \sigma)$-continuous;

(b) $f^{-1}(V)$ is $\mu$-open for each $\theta$-open set $V$ of $Y$;

(c) $f^{-1}(F)$ is $\mu$-closed for each $\theta$-closed set $F$ of $Y$.

Proof. (a) $\Rightarrow$ (b): Let $V$ be a $\theta$-open set of $Y$, and let $x \in$ $f^{-1}(V)$. Since $f(x) \in V$ and $f$ is faintly $(\mu, \sigma)$-continuous, there exists $U \in \mu$ containing $x$ such that $f(U) \subseteq V$. It then follows that $x \in U \subseteq f^{-1}(V)$. Hence, $f^{-1}(V)$ is $\mu$-open.

(b) $\Rightarrow$ (a): Let $x \in X$ and let $V$ be a $\theta$-open set in $Y$ containing $f(x)$. Then, by (b), $f^{-1}(V)$ is $\mu$-open in $X$ containing $x$. Let $U=f^{-1}(V)$. Then, $f(U) \subseteq V$.

(b) $\Rightarrow$ (c): Let $F$ be a $\theta$-closed set of $Y$. Since $Y \backslash F$ is $\theta$ open, by (b), it follows that $f^{-1}(Y \backslash F)=X \backslash f^{-1}(F)$ is $\mu$-open. This shows that $f^{-1}(F)$ is $\theta$-closed.

(c) $\Rightarrow$ (b): Let $V$ be a $\theta$-open set in $Y$. Then, $Y \backslash V$ is $\theta$ closed in $Y$. By (c), $f^{-1}(Y \backslash V)=X \backslash f^{-1}(V)$ is $\theta$-closed. Thus, $f^{-1}(V)$ is $\mu$-open.

Theorem 6. If a function $f:(X, \mu) \rightarrow(Y, \sigma)$ is weakly $(\mu, \sigma)$ continuous, then it is faintly $(\mu, \sigma)$-continuous.

Proof. Let $x \in X$, and let $V$ be a $\theta$-open set containing $f(x)$. Then, there exists an open set $W$ such that $f(x) \in W \subseteq$ $\operatorname{cl}(W) \subseteq V$. Since $f$ is weakly $(\mu, \sigma)$-continuous, there exists a $\mu$-open set $U$ containing $x$ such that $f(U) \subseteq \operatorname{cl}(W) \subseteq V$. Thus, $f$ is faintly $(\mu, \sigma)$-continuous.

Example 7. Let $X=Y=\{a, b, c\}$, let $\mu=\{\varnothing,\{a\},\{a, b\}, X\}$, and let $\sigma=\{\varnothing,\{a\},\{b\},\{a, b\}, X\}$. The identity function $f$ : $(X, \mu) \rightarrow(Y, \sigma)$ is faintly $(\mu, \sigma)$-continuous but not weakly $(\mu, \sigma)$-continuous.

Definition 8. A function $f:(X, \mu) \rightarrow(Y, \sigma)$ is called slightly $(\mu, \sigma)$-continuous if for each $x \in X$ and each clopen set $V$ of $Y$ containing $f(x)$ there exists a $\mu$-open set $U$ containing $x$ such that $f(U) \subseteq V$.

Theorem 9. If $f:(X, \mu) \rightarrow(Y, \sigma)$ is faintly $(\mu, \sigma)$-continuous, then it is slightly $(\mu, \sigma)$-continuous.

Proof. Let $x \in X$, and let $V$ be a clopen set containing $f(x)$. Then, $V$ is $\theta$-open. Since $f$ is faintly $(\mu, \sigma)$-continuous, there exists $U \in \mu$ containing $x$ such that $f(U) \subseteq V$ showing $f$ to be slightly $(\mu, \sigma)$-continuous.

Example 10. Let $\mathbb{R}$ be the set of real numbers. Consider the identity mapping $f:\left(\mathbb{R}, \tau_{\text {co }}\right) \rightarrow\left(\mathbb{R}, \tau_{u}\right)$, where $\tau_{\text {co }}$ and $\tau_{u}$ denote the cocountable and usual topology, respectively. It is easy to show that $f$ is slightly $(\mu, \sigma)$-continuous but not faintly $(\mu, \sigma)$-continuous. 
Remark 11. From Remark 3 and Theorems 6 and 9, we have the following implications:

$(\mu, \sigma)$-continuity $\Rightarrow$ almost $(\mu, \sigma)$-continuity $\Rightarrow$ weakly $(\mu, \sigma)$-continuity $\Rightarrow$ faintly $(\mu, \sigma)$-continuity $\Rightarrow$ slightly $(\mu, \sigma)$-continuity.

Theorem 12. Let $(Y, \sigma)$ be regular. If a function $f:(X, \mu) \rightarrow$ $(Y, \sigma)$ is faintly $(\mu, \sigma)$-continuous, then it is $(\mu, \sigma)$-continuous.

Proof. Let $V$ be an open set of $Y$ containing $f(x)$. Since $Y$ is regular, $V$ is $\theta$-open in $Y$. Then by Theorem $5, f^{-1}(V)$ is $\mu$ open in $X$. Let $U=f^{-1}(V)$. Then, $U$ is a $\mu$-open set containing $x$ such that $f(U) \subseteq V$. Thus, $f$ is $(\mu, \sigma)$-continuous.

Definition 13. A topological space $(Y, \sigma)$ is said to be almost regular [31] if for any regular closed set $F$ and any point $x \in$ $Y \backslash F$ there exist disjoint open sets $U$ and $V$ such that $x \in U$ and $F \subseteq V$.

Theorem 14. If a function $f:(X, \mu) \rightarrow(Y, \sigma)$ is faintly $(\mu, \sigma)$ continuous and $(Y, \sigma)$ is almost regular, then $f$ is almost $(\mu, \sigma)$ continuous.

Proof. Let $x \in X$, and let $V$ be an open set in $Y$. Then, $\operatorname{int}(\mathrm{cl}(V))$ is a regular open set in $Y$ so that it is $\theta$-open in $Y$ (as in an almost regular space, every regular open set is $\theta$-open [20]). Hence, by faintly $(\mu, \sigma)$-continuity of $f$, there exists a $\mu$ open set $U$ containing $x$ such that $f(U) \subseteq \operatorname{int}(\operatorname{cl}(V))$ showing $f$ to be almost $(\mu, \sigma)$-continuous.

The clopen subsets of a topological space $(X, \tau)$ form a base for a topology on $X$. This topology is called ultraregularization [32] of $\tau$ and is denoted by $\tau_{u}$. A topological space $(X, \tau)$ is said to be ultra-regular [33] if $\tau=\tau_{u}$.

Theorem 15. If $(Y, \sigma)$ is ultraregular, then for a function $f$ : $(X, \mu) \rightarrow(Y, \sigma)$ the following are equivalent:

(i) $f$ is $(\mu, \sigma)$-continuous;

(ii) $f$ is almost $(\mu, \sigma)$-continuous;

(iii) $f$ is weakly $(\mu, \sigma)$-continuous;

(iv) $f$ is faintly $(\mu, \sigma)$-continuous;

(v) $f$ is slightly $(\mu, \sigma)$-continuous.

Proof. We will only show that in an ultra regular space every slightly $(\mu, \sigma)$-continuous function is $(\mu, \sigma)$-continuous. The rest will follow from Remark 11. Let $V$ be an open set in $Y$ containing $f(x)$. Then, as $(Y, \sigma)$ is ultra regular, there exists a clopen set $W$ in $Y$ containing $f(x)$ such that $W \subseteq V$. Since $f$ is slightly $(\mu, \sigma)$-continuous, there exists a $\mu$-open set $U$ in $X$ containing $x$ such that $f(U) \subseteq W \subseteq V$. Thus, $f$ is $(\mu, \sigma)$ continuous.

Theorem 16. If $\mu$ and $\lambda$ are two GTs on $X$ such that $\mu \subseteq \lambda$ and if $f:(X, \mu) \rightarrow(Y, \sigma)$ is faintly $(\mu, \sigma)$-continuous, then $f$ is faintly $(\lambda, \sigma)$-continuous.

Proof. It follows immediately from Theorem 5.
Observation. Let $(X, \tau)$ be a topological space. Then, we have

(i) $\tau \subseteq \alpha O(X) \subseteq P O(X) \subseteq B O(X) \subseteq \beta O(X)$;

(ii) $\tau \leqq \alpha O(X) \subseteq P O(X) \subseteq \delta P O(X) \subseteq e O(X)$;

(iii) $\tau \subseteq \alpha O(X) \subseteq S O(X) \subseteq B O(X) \subseteq \beta O(X)$;

(iv) $\tau \subseteq \alpha O(X) \subseteq S O(X) \subseteq e O(X)$;

(v) $P_{\theta} O(X) \subseteq P O(X), \theta O(X) \subseteq S_{\theta} O(X) \subseteq \delta S O(X) \subseteq$ $S O(X)$.

Thus, from Theorem 16, we can deduce relations between different types of faintly $(\mu, \sigma)$-continuous functions.

\section{Properties of Faintly $(\mu, \sigma)$-Continuous Functions}

Definition 17. A GTS $(X, \mu)$ (resp., a topological space $(X, \tau))$ is said to be $\mu-T_{2}$ [34] (resp., $\theta-T_{2}$ [35]) if for any two distinct points $x, y$ of $X$ there exist two disjoint $\mu$-open (resp., $\theta$-open) sets $U$ and $V$ containing $x$ and $y$, respectively.

It is well known from [36] that a topological space $(X, \tau)$ is Hausdorff if and only if it is $\theta-T_{2}$.

Theorem 18. If $f:(X, \mu) \rightarrow(Y, \sigma)$ is a faintly $(\mu, \sigma)$-continuous injection and $(Y, \sigma)$ is $T_{2}$, then $(X, \mu)$ is $\mu-T_{2}$.

Proof. Let $x$ and $y$ be two distinct points of $X$. Then, $f(x)$ and $f(y)$ are two distinct points of $Y$. Thus, there exist two disjoint open sets $U$ and $V$ containing $x$ and $y$ respectively. Then, by Theorem $5, f^{-1}(U)$ and $f^{-1}(V)$ are two $\mu$-open sets in $X$. Clearly, $x \in f^{-1}(U), y \in f^{-1}(V)$, and $f^{-1}(U) \cap f^{-1}(V)=$ $\varnothing$ showing $(X, \mu)$ to be $\mu-T_{2}$.

Definition 19. A topological space $(X, \tau)$ is said to be

(i) $\theta$-regular [26] if for each $\theta$-closed set $F$ and each point $x \notin F$ there exist disjoint $\theta$-open sets $U$ and $V$ such that $x \in U, F \subseteq V$;

(ii) $\theta$-normal [26] if for any two disjoint $\theta$-closed subsets $F$ and $K$ there exist disjoint $\theta$-open sets $U$ and $V$ such that $F \subseteq U, K \subseteq V$.

Definition 20. A GTS $(X, \mu)$ is said to be

(i) $\mu$-regular [37] if for each $\mu$-closed set $F$ and each point $x \notin F$, there exist disjoint $\mu$-open sets $U$ and $V$ such that $x \in U, F \subseteq V$.

(ii) $\mu$-normal [37] if for any two disjoint $\mu$-closed subsets $F$ and $K$, there exist disjoint $\mu$-open sets $U$ and $V$ such that $F \leqq U, K \subseteq V$.

Definition 21. A function $f:(X, \mu) \rightarrow(Y, \sigma)$ is called $\left(\mu, \sigma_{\theta}\right)$ open if for each $\mu$-open set $V$ in $X, f(V)$ is $\theta$-open in $(Y, \sigma)$.

Theorem 22. If $f:(X, \mu) \rightarrow(Y, \sigma)$ is faintly $(\mu, \sigma)$-continuous, $\left(\mu, \sigma_{\theta}\right)$-open bijection, and $(X, \mu)$ is $\mu$-regular, then $(Y, \sigma)$ is $\theta$-regular. 
Proof. Let $F$ be a $\theta$-closed subset of $Y$, and let $y \notin F$. Let $y=f(x)$. Since $f$ is faintly $(\mu, \sigma)$-continuous, by Theorem 5 , $f^{-1}(F)$ is $\mu$-closed in $X$ so that $f^{-1}(y)=x \notin f^{-1}(F)$. Let $G=f^{-1}(F)$. Then, $x \notin G$; thus, by $\mu$-regularity of $(X, \mu)$, there exist two disjoint $\mu$-open sets $U$ and $V$ such that $G \subseteq U$ and $x \in V$. Thus, we have $F=f(G) \subseteq f(U)$ and $y=f(x) \in f(V)$ and $f(U) \cap f(V)=\varnothing$. As $f$ is $\left(\mu, \sigma_{\theta}\right)$-open, $f(U)$ and $f(V)$ are $\theta$-open in $(Y, \sigma)$ showing $(Y, \sigma)$ to be $\theta$-regular.

Theorem 23. If $f:(X, \mu) \rightarrow(Y, \sigma)$ is faintly $(\mu, \sigma)$-continuous, $\left(\mu, \sigma_{\theta}\right)$-open surjection, and $(X, \mu)$ is $\mu$-normal, then $(Y, \sigma)$ is $\theta$-normal.

Proof. Let $F_{1}$ and $F_{2}$ be two disjoint $\theta$-closed subsets of $(Y, \sigma)$. Since $f$ is faintly $(\mu, \sigma)$-continuous, by Theorem $5, f^{-1}\left(F_{1}\right)$ and $f^{-1}\left(F_{2}\right)$ are two $\mu$-closed subsets in $X$. Let $K_{1}=f^{-1}\left(F_{1}\right)$, and let $K_{2}=f^{-1}\left(F_{2}\right)$. Then, $K_{1}$ and $K_{2}$ are two disjoint $\mu$ closed subsets of $(X, \mu)$. Since $(X, \mu)$ is $\mu$-normal, there exist two disjoint $\mu$-open sets $U$ and $V$ such that $K_{1} \subseteq U$ and $K_{2} \subseteq$ $V$. We thus have $F_{1}=f\left(K_{1}\right) \subseteq f(U)$ and $F_{2}=f\left(K_{2}\right) \subseteq f(V)$. Also $f(U)$ and $f(V)$ are two disjoint $\theta$-open sets in $(Y, \sigma)$ showing $(Y, \sigma)$ to be $\theta$-normal.

Definition 24. A GTS $(X, \mu)$ is said to be $\mu$-connected [38] if $X$ cannot be written as union of two nonempty $\mu$-open sets.

Theorem 25. If $f:(X, \mu) \rightarrow(Y, \sigma)$ is a faintly $(\mu, \sigma)$-continuous surjection and $(X, \mu)$ is $\mu$-connected, then $(Y, \sigma)$ is connected.

Proof. Let us assume that $(Y, \sigma)$ be not connected. Then, there exist nonempty disjoint open sets $V_{1}$ and $V_{2}$ such that $Y=$ $V_{1} \cup V_{2}$. Hence, we have $f^{-1}\left(V_{1}\right) \cup f^{-1}\left(V_{2}\right)=X$ and $f^{-1}\left(V_{1}\right) \cap$ $f^{-1}\left(V_{2}\right)=\varnothing$. Since $f$ is surjective, $f^{-1}\left(V_{1}\right)$ and $f^{-1}\left(V_{2}\right)$ are nonempty. Since $V_{1}$ and $V_{2}$ are clopen, they are $\theta$-open in $Y$. Thus by Theorem $5, f^{-1}\left(V_{1}\right)$ and $f^{-1}\left(V_{2}\right)$ are $\mu$-open. Therefore $(X, \mu)$ is not $\mu$-connected-a contradiction.

Definition 26. A GTS $(X, \mu)$ is called $\mu$-compact [39] if every $\mu$-open cover of $X$ has a finite subcover. A subset $A$ of $X$ is said to be $\mu$-compact relative to $(X, \mu)$ if every cover of $A$ by $\mu$-open sets of $X$ has a finite subcover.

Definition 27. A subset $A$ of a topological space $(Y, \sigma)$ is called $\theta$-compact relative to $(Y, \sigma)[40]$ if every cover of $A$ by $\theta$-open sets of $(Y, \sigma)$ has a finite subcover. A topological space $(Y, \sigma)$ is called $\theta$-compact if $Y$ is $\theta$-compact relative to $(Y, \sigma)$.

Theorem 28. If $f:(X, \mu) \rightarrow(Y, \sigma)$ is a faintly $(\mu, \sigma)$-continuous function and $A$ is $\mu$-compact relative to $(X, \mu)$, then $f(A)$ is $\theta$-compact relative to $(Y, \sigma)$.

Proof. Let $\left\{V_{\alpha}: \alpha \in \Lambda\right\}$ be a cover of $f(A)$ by $\theta$-open sets of $Y$. Then, for each $x \in A$, there exists $\alpha_{x} \in \Lambda$ such $f(x) \in V_{\alpha_{x}}$. Since $f$ is a faintly $(\mu, \sigma)$-continuous function, there exists $U_{x} \in \mu$ containing $x$ such that $f\left(U_{x}\right) \subseteq V_{\alpha_{x}}$. Then, the family $\left\{U_{x}: x \in A\right\}$ is a cover of $A$ by $\mu$-open sets of $(X, \mu)$. Since $A$ is $\mu$-compact relative to $(X, \mu)$, there exists a finite number of points, say, $x_{1}, x_{2}, \ldots, x_{n} \in A$, such that $A \subseteq \cup\left\{U_{x_{i}}: i=\right.$ $\left.1,2, \ldots, n, x_{i} \in A\right\}$. Therefore, we have $f(A) \subseteq \cup\left\{f\left(U_{x_{i}}\right)\right.$ : $\left.i=1,2, \ldots, n, x_{i} \in A\right\} \subseteq \cup\left\{V_{\alpha_{\left(x_{i}\right)}}: i=1,2, \ldots, n, x_{i} \in A\right\}$. This shows that $f(A)$ is $\theta$-compact relative to $(Y, \sigma)$.

Definition 29. For any subset $A$ of a $\operatorname{GTS}(X, \mu)$, the $\mu$-frontier of $A$ is denoted by $\operatorname{Fr}_{\mu}(A)$ and defined by $\operatorname{Fr}_{\mu}(A)=c_{\mu}(A) \cap$ $c_{\mu}(X \backslash A)$.

Theorem 30. The set of all points $x \in X$ at which the function $f:(X, \mu) \rightarrow(Y, \sigma)$ is not faintly $(\mu, \sigma)$-continuous is identical with the union of $\mu$-frontier of the inverse images of $\theta$-open sets of $(Y, \sigma)$ containing $f(x)$.

Proof. Suppose that $f$ is not faintly $(\mu, \sigma)$-continuous at $x \in$ $X$. Then, there exists a $\theta$-open set $V$ of $Y$ containing $f(x)$ such that $f(U)$ is not a subset of $V$ for each $\mu$-open set $U$ containing $x \in X$. Hence, we have $U \cap\left(X \backslash f^{-1}(V)\right) \neq \varnothing$ for each $U \in \mu$ containing $x$. So, $x \in c_{\mu}\left(X \backslash f^{-1}(V)\right)$. On the other hand, we have $x \in f^{-1}(V) \subseteq c_{\mu}\left(f^{-1}(V)\right)$. Hence $x \in \operatorname{Fr}_{\mu}\left(f^{-1}(V)\right)$.

Conversely, suppose that $f$ is not faintly $(\mu, \sigma)$-continuous at $x \in X$, and let $V$ be any $\theta$-open set containing $f(x)$. Then, by Theorem $5, f^{-1}(V)=i_{\mu}\left(f^{-1}(V)\right)$. Therefore, $x \notin \operatorname{Fr}_{\mu}\left(f^{-1}(V)\right)$ for each $\theta$-open set $V$ containing $f(x)$. This completes the proof.

\section{Faintly $\mu$-Closed Graph}

Definition 31. A function $f:(X, \mu) \rightarrow(Y, \sigma)$ is said to have a faintly $\mu$-closed graph if for each $(x, y) \in(X \times Y) \backslash G(f)$ there exist $U \in \mu$ containing $x$ and a $\theta$-open set $V$ in $Y$ containing $y$ such that $(U \times V) \cap G(f)=\varnothing$.

Lemma 32. The graph $G(f)$ of a function $f:(X, \mu) \rightarrow(Y, \sigma)$ is faintly $\mu$-closed if and only if for each $(x, y) \in(X \times Y) \backslash$ $G(f)$ there exist $U \in \mu$ containing $x$ and $a \theta$-open set $V$ in $Y$ containing $y$ such that $f(U) \cap V=\varnothing$.

Theorem 33. Let a function $f:(X, \mu) \rightarrow(Y, \sigma)$ have a faintly $\mu$-closed graph $G(f)$. If $f$ is a faintly $(\mu, \sigma)$-continuous injection, then $(X, \mu)$ is $\mu-T_{2}$.

Proof. Let $x$ and $y$ be two distinct points of $X$. Then, since $f$ is an injection, we have $f(x) \neq f(y)$. Then, we have $(x, f(y)) \in$ $X \times Y \backslash G(f)$. Thus, by Lemma 32, there exist a $\mu$-open set $U$ containing $x$ in $X$ and a $\theta$-open set $V$ in $Y$ containing $f(y)$ such that $f(U) \cap V=\varnothing$. Hence, $U \cap f^{-1}(V)=\varnothing$, and $y \notin$ $U$. Since $f$ is faintly $(\mu, \sigma)$-continuous, there exists a $\mu$-open set $W$ containing $y$ such that $f(W) \subseteq V$. Therefore, we have $f(U) \cap f(W)=\varnothing$. Since $f$ is injective, we obtain $U \cap W=\varnothing$ showing $(X, \mu)$ to be $\mu-T_{2}$.

Definition 34. A function $f:(X, \mu) \rightarrow(Y, \sigma)$ is said to have a faintly strong $\mu$-closed graph if for each $(x, y) \in(X \times Y) \backslash$ $G(f)$ there exist $U \in \mu$ containing $x$ and an open set $V$ in $Y$ containing $y$ such that $(U \times \operatorname{cl}(V)) \cap G(f)=\varnothing$.

Lemma 35. The graph $G(f)$ of a function $f:(X, \mu) \rightarrow(Y, \sigma)$ is faintly strong $\mu$-closed if and only if for each $(x, y) \in(X \times$ $Y) \backslash G(f)$ there exist $U \in \mu$ containing $x$ and an open set $V$ in $Y$ containing $y$ such that $f(U) \cap \mathrm{cl}(V)=\varnothing$. 
Theorem 36. If a function $f:(X, \mu) \rightarrow(Y, \sigma)$ is faintly $(\mu, \sigma)$-continuous and $(Y, \sigma)$ is $T_{2}$, then $G(f)$ is faintly strong $\mu$-closed.

Proof. Let $x \in X$, and let $y=f(x)$. Then, $(x, y) \in(X \times Y) \backslash$ $G(f)$. Then, by Lemma 32, there exist a $\mu$-open set $U$ in $X$ containing $x$ and a $\theta$-open set $V$ in $Y$ containing $y$ such that $f(U) \cap V=\varnothing$. Since $V$ is $\theta$-open, there exists an open set $V_{0}$ in $Y$ containing $y$ such that $y \in V_{0} \subseteq \operatorname{cl}\left(V_{0}\right) \subseteq V$. Thus $f(U) \cap \mathrm{cl}\left(V_{0}\right)=\varnothing$. Thus, by Lemma $35, G(f)$ is faintly strong $\mu$-closed.

Theorem 37. If a function $f:(X, \mu) \rightarrow(Y, \sigma)$ is a surjective function with faintly strong $\mu$-closed graph $G(f)$, then $(Y, \sigma)$ is $T_{2}$.

Proof. Let $y_{1}$ and $y_{2}$ be any two distinct points of $Y$. Then, since $f$ is surjective, there exists $x_{1} \in X$ such that $f\left(x_{1}\right)=y_{1}$; hence, $\left(x_{1}, y_{2}\right) \in(X \times Y) \backslash G(f)$. Since $G(f)$ is faintly strong $\mu$-closed, there exist a $\mu$-open set $U$ in $X$ containing $x_{1}$ and an open set $V$ of $Y$ containing $y_{2}$ such that $f(U) \cap \operatorname{cl}(V)=\varnothing$. Therefore, we have $y_{1}=f\left(x_{1}\right) \in f(U) \subseteq Y \backslash \mathrm{cl}(V)$. Hence, there exists an open set $H$ of $Y$ such that $y_{1} \in H$ and $H \cap V=$ $\varnothing$. Moreover, we have $y_{2} \in V$, and $V$ is open in $Y$. This shows that $(Y, \sigma)$ is $T_{2}$.

Theorem 38. If a function $f:(X, \mu) \rightarrow(Y, \sigma)$ has a faintly $\mu$-closed graph, then it has also a faintly strong $\mu$-closed graph.

Proof. Let $x \in X$, and let $y \neq f(x)$. Then, $(x, y) \in(X \times Y) \backslash$ $G(f)$. Then there exist $U$ in $\mu$ containing $x$ in $X$ and a $\theta$-open set $V$ in $Y$ containing $y$ such that $f(U) \cap V=\varnothing$. Since $V$ is $\theta$-open, there exists an open set $V_{0}$ such that $V_{0} \subseteq \operatorname{cl}\left(V_{0}\right) \subseteq V$. So $f(U) \cap \operatorname{cl}\left(V_{0}\right)=\varnothing$. Thus, by Lemma $35, f$ has a faintly strong $\mu$-closed graph.

The converse of the above theorem is not true in general as shown in Example 3 of [20].

Theorem 39. If $f:(X, \mu) \rightarrow(Y, \sigma)$ has a faintly $\mu$-closed graph, then $f(K)$ is closed in $(Y, \sigma)$ for each subset $K$ which is $\mu$-compact relative to $(X, \mu)$.

Proof. Suppose that $y \notin f(K)$. Then, for each $x \in K,(x, y) \notin$ $G(f)$. Since $G(f)$ is faintly $\mu$-closed, by Lemma 32, there exist a $\mu$-open set $U_{x}$ in $X$ containing $x$ and a $\theta$-open set $V_{x}$ in $Y$ containing $y$ such that $f\left(U_{x}\right) \cap V_{x}=\varnothing$. Then, the family $\left\{U_{x}: x \in K\right\}$ is a cover of $K$ by $\mu$-open sets in $X$. So, there exists a finite subfamily $K_{0}$ of $K$ such that $K \subseteq \cup\left\{U_{x}: x \in K_{0}\right\}$. Set $V=\cap\left\{V_{x}: x \in K_{0}\right\}$. Then, $V$ is $\theta$-open (hence open) in $Y$ containing $y$. Therefore, $f(K) \cap V \subseteq \cup\left\{f\left(U_{x}\right): x \in K_{0}\right\} \cap$ $V \subseteq \cup\left\{f\left(U_{x}\right) \cap V: x \in K_{0}\right\}=\varnothing$. It then follows that $y \notin$ $\operatorname{cl}(f(K))$. Thus, $f(K)$ is closed in $Y$.

\section{Conclusion}

Similar types of faintly continuous functions can be defined from a topological space $(X, \tau)$ to another topological space $(Y, \sigma)$ from the definition of faintly $(\mu, \sigma)$-continuous function by taking different GTs on $X$. In fact, different results on weak forms of faintly continuous functions can be derived from faintly $(\mu, \sigma)$-continuous functions by replacing $\mu$ by the corresponding GTs on $X$ (see [20-29]).

\section{Conflict of Interests}

The author Bishwambhar Roy declares that the paper does not have any financial relation with any commercial identity that might lead to conflict of interests.

\section{Acknowledgment}

The author is thankful to the referee for his/her comments to improve the paper.

\section{References}

[1] Á. Császár, “Generalized topology, generalized continuity," Acta Mathematica Hungarica, vol. 96, no. 4, pp. 351-357, 2002.

[2] Á. Császár, "Generalized open sets in generalized topologies," Acta Mathematica Hungarica, vol. 106, no. 1-2, pp. 53-66, 2005.

[3] Á. Császár, " $\delta$ - and $\theta$-modifications of generalized topologies," Acta Mathematica Hungarica, vol. 120, no. 3, pp. 275-279, 2008.

[4] Á. Császár, "Separation axioms for generalized topologies," Acta Mathematica Hungarica, vol. 104, no. 1-2, pp. 63-69, 2004.

[5] Á. Császár, “ $\gamma$-connected sets," Acta Mathematica Hungarica, vol. 101, no. 4, pp. 273-279, 2003.

[6] N. V. Veličko, "H-closed topological spaces," Matematicheskii Sbornik, vol. 70, no. 112, pp. 98-112, 1966.

[7] N. Levine, "Semi-open sets and semi-continuity in topological spaces," The American Mathematical Monthly, vol. 70, pp. 36-41, 1963.

[8] A. S. Mashhour, M. E. Abd El-Monsef, and S. N. El-Deep, "On precontinuous and weak precontinuous mappings," Proceedings of the Mathematical and Physical Society of Egypt, vol. 53, pp. 4753, 1982.

[9] O. Njästad, “On some classes of nearly open sets," Pacific Journal of Mathematics, vol. 15, pp. 961-970, 1965.

[10] M. E. Abd El-Monsef, S. N. El-Deeb, and R. A. Mahmoud, “ $\beta$ open sets and $\beta$-continuous mapping," Bulletin of the Faculty of Science. Assiut University A, vol. 12, no. 1, pp. 77-90, 1983.

[11] D. Andrijević, "Semi-preopen sets," Matematički Vesnik, vol. 38, pp. 24-32, 1986.

[12] S. Raychaudhuri and M. N. Mukherjee, "On $\delta$-almost continuity and $\delta$-preopen sets," Bulletin of the Institute of Mathematics. Academia Sinica, vol. 21, no. 4, pp. 357-366, 1993.

[13] J. H. Park, D. S. Song, and R. Saadati, "On generalized $\delta$-semiclosed sets in topological spaces," Chaos, Solitons and Fractals, vol. 33, no. 4, pp. 1329-1338, 2007.

[14] E. Ekici, "On $e$-open sets DP* -sets DPE* -sets and decompositions of continuity," The Arabian Journal for Science and Engineering $A$, vol. 33, no. 2, pp. 269-282, 2008.

[15] G. Di Maio and T. Noiri, "On $s$-closed spaces," Indian Journal of Pure and Applied Mathematics, vol. 18, no. 3, pp. 226-233, 1987.

[16] M. C. Pal and P. Bhattacharyya, "Feeble and strong forms of preirresolute functions," Bulletin of the Malaysian Mathematical Sciences Society, vol. 19, no. 2, pp. 63-75, 1996.

[17] J. Dontchev and T. Noiri, "Quasi-normal spaces and $\pi g$-closed sets," Acta Mathematica Hungarica, vol. 89, no. 3, pp. 211-219, 2000 . 
[18] H. Z. Hdeib, “ $\omega$-closed mappings," Revista Colombiana de Matemáticas, vol. 16, no. 1-2, pp. 65-78, 1982.

[19] B. Roy, "Unification of almost strongly $\mu_{\theta}$-continuous functions," accepted in Le Mathematiche.

[20] P. E. Long and L. L. Herrington, "The $T_{\theta}$-topology and faintly continuous functions," Kyungpook Mathematical Journal, vol. 22, no. 1, pp. 7-14, 1982.

[21] T. Noiri and V. Popa, "Weak forms of faint continuity," Bulletin Mathématique de la Société des Sciences Mathématiques de la République Socialiste de Roumanie, vol. 34, no. 3, pp. 263-270, 1990.

[22] S. Jafari and T. Noiri, "On faintly $\alpha$-continuous functions," Indian Journal of Mathematics, vol. 42, no. 2, pp. 203-210, 2000.

[23] A. A. Nasef, "Another weak form of faint continuity," Chaos, Solitons and Fractals, vol. 12, no. 12, pp. 2219-2225, 2001.

[24] E. Ekici, "On almost continuity," Kyungpook Mathematical Journal, vol. 46, no. 1, pp. 119-130, 2006.

[25] E. Ekici, "On $\delta$-semiopen sets and a generalization of functions," Boletim da Sociedade Paranaense de Matemática, vol. 23, no. 1-2, pp. 73-84, 2005.

[26] M. C. Caldas, "On the faintly $e$-continuous functions," Sarajevo Journal of Mathematics, vol. 8, no. 1, pp. 159-170, 2012.

[27] A. A. El-Atik, "On some types of faint continuity," Thai Journal of Mathematics, vol. 9, no. 1, pp. 83-93, 2011.

[28] N. Rajesh, "On faintly $\pi g$-continuous functions," Boletim da Sociedade Paranaense de Matemática, vol. 30, no. 1, pp. 9-19, 2012.

[29] E. Ekici, S. Jafari, and S. P. Moshokoa, "On a weaker form of $\omega$-continuity," Annals of the University of Craiova. Mathematics and Computer Science Series, vol. 37, no. 2, pp. 38-46, 2010.

[30] T. Noiri and V. Popa, "Faintly $m$-continuous functions," Chaos, Solitons and Fractals, vol. 19, no. 5, pp. 1147-1159, 2004.

[31] M. K. Singal and S. Prabha Arya, "On almost-regular spaces," Glasnik Matematički, vol. 4, no. 24, pp. 89-99, 1969.

[32] T. Noiri, "Slightly $\beta$-continuous functions," International Journal of Mathematics and Mathematical Sciences, vol. 28, no. 8, pp. 469-478, 2001.

[33] R. L. Ellis, "A non-Archimedean analogue of the Tietze-Urysohn extension theorem," Indagationes Mathematicae, vol. 29, pp. 332-333, 1967.

[34] M. S. Sarsak, "Weak separation axioms in generalized topological spaces," Acta Mathematica Hungarica, vol. 131, no. 1-2, pp. 110-121, 2011.

[35] S. Sinharoy and S. Bandyopadhyay, "On $\theta$-completely regular and locally $\theta-H$-closed spaces," Bulletin of the Calcutta Mathematical Society, vol. 87, no. 1, pp. 19-26, 1995.

[36] M. C. Caldas, S. Jafari, and T. Noiri, "Some separation axioms via modified $\theta$-open sets," Bulletin of the Iranian Mathematical Society, vol. 29, no. 2, pp. 1-12, 2003.

[37] B. Roy, "On a type of generalized open sets," Applied General Topology, vol. 12, no. 2, pp. 163-173, 2011.

[38] R.-X. Shen, "A note on generalized connectedness," Acta Mathematica Hungarica, vol. 122, no. 3, pp. 231-235, 2009.

[39] B. Roy and S. Jafari, "On covering properties via generalized open sets," Annales Universitatis Scientiarum Budapestinensis de Rolando Eötvös Nominatae. Mathematica, vol. 55, pp. 57-65, 2012.

[40] S. Jafari, "Some properties of quasi $\theta$-continuous functions," Far East Journal of Mathematical Sciences, vol. 6, no. 5, pp. 689-696, 1998. 


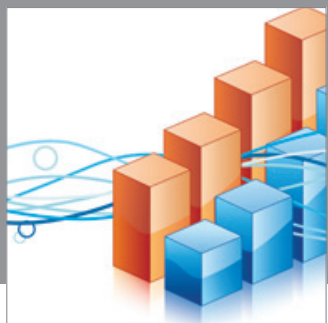

Advances in

Operations Research

mansans

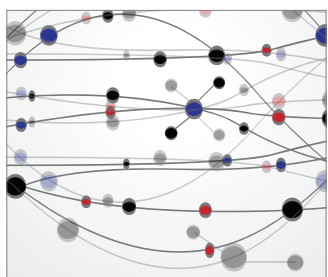

The Scientific World Journal
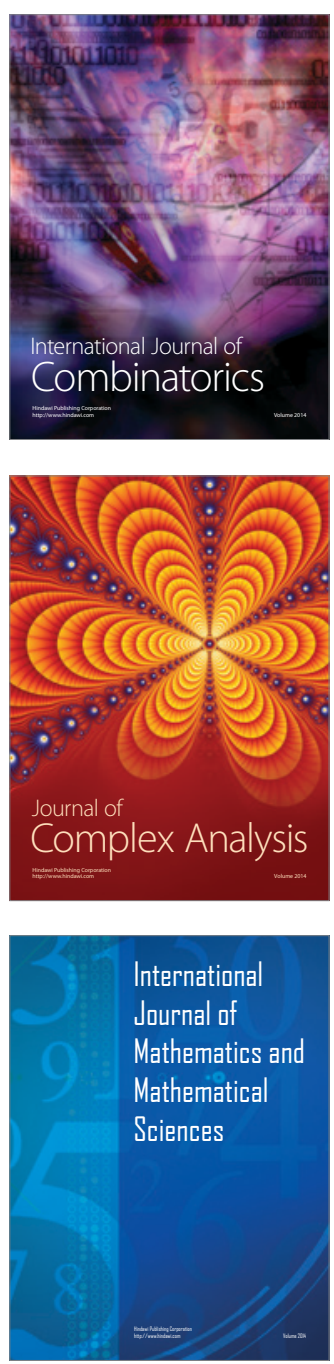
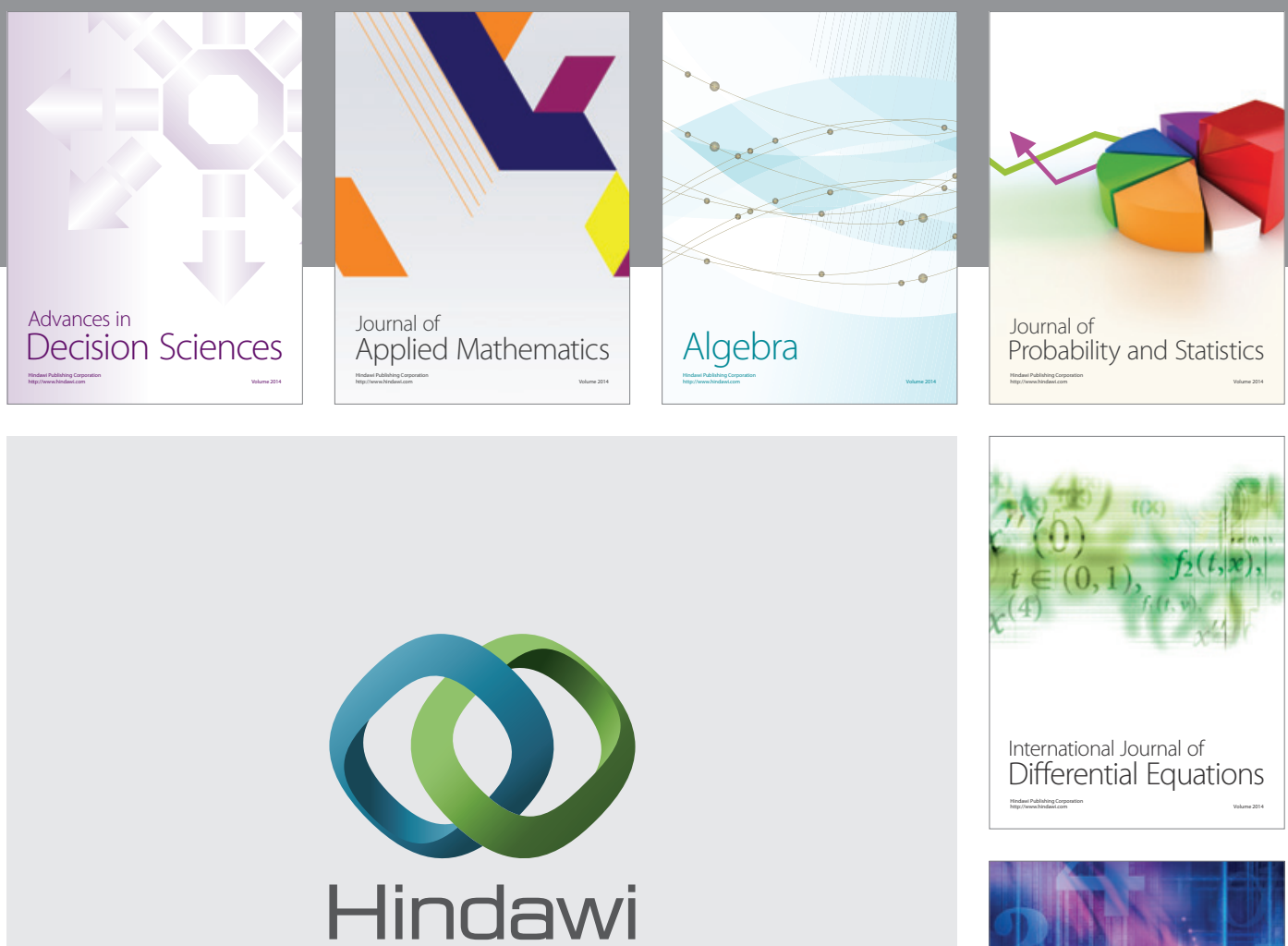

Submit your manuscripts at http://www.hindawi.com
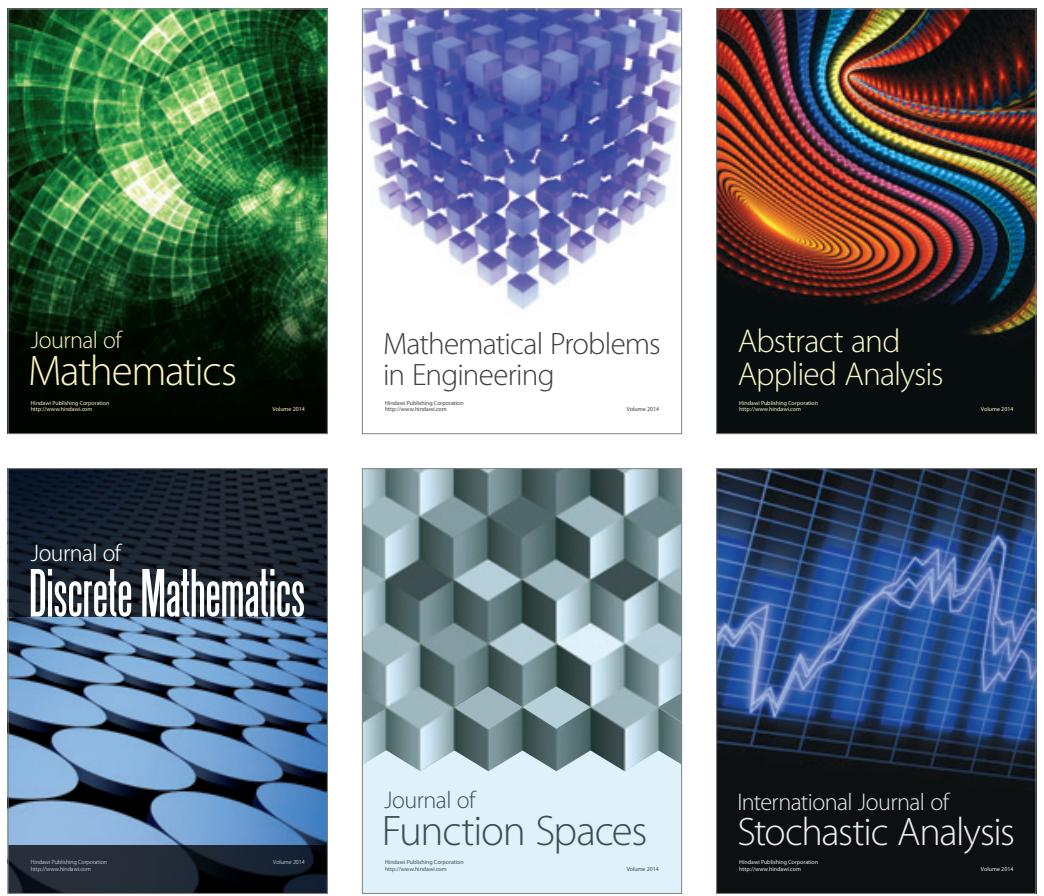

Journal of

Function Spaces

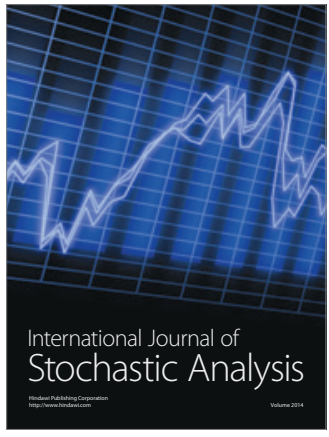

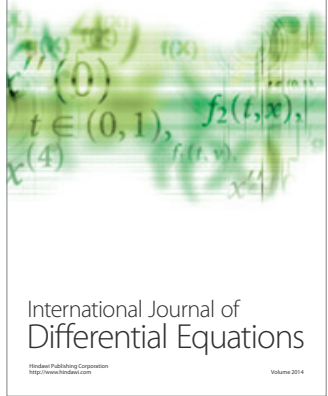
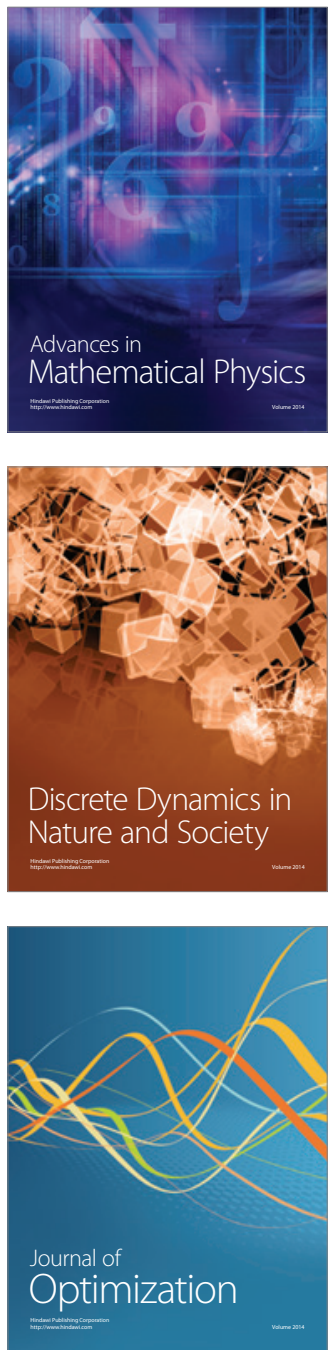